222 Bamberger u.deGruyter: Ueb.Formazylmethylketon.

\title{
Ueber Formazylmethylketon;
}

\section{von \\ Eugen Bamberger und Paul de Gruyter.}

Die Untersuchungen über diesen Körper, welcher von Bamberger und $\mathrm{Wulz}^{1}$ ) gelegentlich einer Untersuchung über die Einwirkung von Diazobenzol auf Aceton entdeckt wurde:

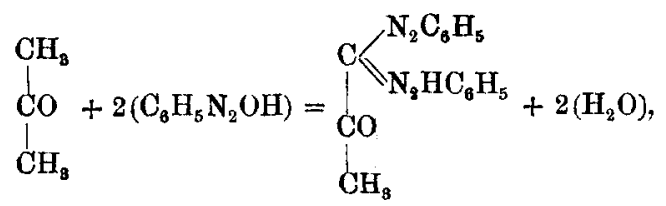

sind, was die allgemeinen Ergebnisse betrifft, schon vor längerer Zeit in Gemeinschaft mit den Herren Wulz ${ }^{1}$ ), Lorenzen ${ }^{2}$ ) und de Gruyter ${ }^{3}$ ) mitgetheilt worden. Im Folgenden sollen die experimentellen Einzelheiten nachgetragen werden, welche bisher nur in Dissertationen veröffentlicht worden sind; die Betheiligung von Herrn Dr. Lorenzen an den nachfolgend beschriebenen Versuchen ist aus den kurzen Publicationen in den Berichten der Deutschen chemischen Gesellschaft ersichtlich.

$$
\begin{aligned}
& \text { Formazylmethylketon, } \\
& \mathrm{CH}_{3}-\mathrm{CO}-\mathrm{C} \\
& \mathrm{C}_{6} \mathrm{H}_{5} \mathrm{~N}_{2} \mathrm{~N}_{2} \mathrm{HC}_{6} \mathrm{H}_{5},
\end{aligned}
$$

entsteht aus Aceton und Diazobenzol ${ }^{1}$ ), als Nebenprodukt ${ }^{4}$ ) bei der Darstellung des Brenztraubenaldebydphenylhydrazons $\mathrm{CH}_{3}-\mathrm{CO}-\mathrm{CH}: \mathrm{N}_{2} \mathrm{HC}_{6} \mathrm{H}_{5}$, aus letzterem und alkalischem Diazobenzol ${ }^{5}$ ), aus der sogenannten „Phenylazoacetessigsäure“

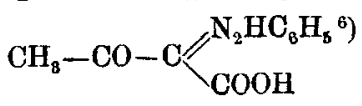
1) Ber. 24, 2793.
2) Das. 25, 3545 .
3) Das. 26, 2783.

4) Das, 24, 3260. Inzwisehen habe ich mich überzengt, dass nicht nur $2-3 \%$, sondern sogar 8-9\% Formazylmethylketon neben dem nach Japp u. Klingemann dargestellten Aldehydrazon entstehen. B.
b) Ber. 24, 3263 .
$\left.{ }^{6}\right)$ Das. 25, 3209. 
Bamberger u.deGruyter: Ueb.Formazylmethylketon. 223

oder auch direct aus A cetessigsäure und Diazobenzol. ${ }^{1}$ ) Den nämlichen Körper erbielt (Claisen ${ }^{2}$ ) aus Acetylaceton und Diazobenzol.

Darstellung aus Phenylazoacetessigsäure und Diazo. benzol ${ }^{3}$,

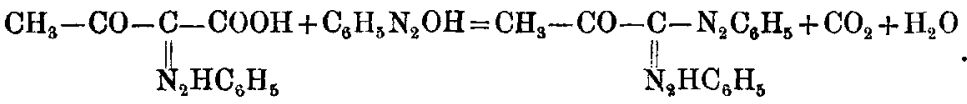

$0,93 \mathrm{Grm}$. Anilin werden diazotirt und in eine eisgekühlte Lösung von 3,6 Grm. wasserfreiem Natriumcarbonat eingetragen. Giesst man diese Diazolösung bei $0^{\circ}$ zu 2 Grm., in verdünnter Soda befindlicher Phenylazoacetessigsäure, so scheidet sich ein rother, harziger, theilweis an der Oberfläche schwimmender Niederschlag $a b$, welcher sich in einigen Minuten in krystallinische, metallisch glänzende Flocken verwandelt. Nach viertelstündigem Stehen in Eis werden dieselben abfiltrirt (1,95 Grm.) und einmal aus siedendem Alkohol umkrystallisirt. Rothe Nadeln, Schmelzp. $134^{\circ}-135^{\circ}$.

$0,1460 \mathrm{Grm}$. Substanz gaben $27,6 \mathrm{Ccm}$. N bei $14^{\circ}$ u. $724,5 \mathrm{Mm}$. B.

Berechnet für $\mathrm{C}_{15} \mathrm{H}_{14} \mathrm{~N}_{4} \mathrm{O}$ :

$$
\mathrm{N}=21,05
$$

Gefunden:

$21,17 \%$.

Am empfehlenswerthesten ist die

Darstellung aus Brenztraubenaldehydphenylhydrazon und Diazobenzol,

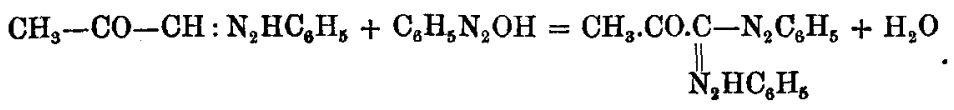

Die auf $0^{0}$ abgekühlte Lösung von $81 \mathrm{Grm}$. rohem Brenztraubenaldehydrazon - man benutze das nach Japp und Klingem $a n^{4}$ ) dargestellte und nicht weiter gereinigte Produkt der Einwirkung von Diazobenzol auf Acetessigsäure - in $1500 \mathrm{Grm}$. Sprit wird mit einer aus $46,5 \mathrm{Grm}$. Anilin bereiteten, concentrirten Diazoniumchloridlösung vermischt und dann unter lebhaftem Rühren in die eisgekühlte Lösung von $250 \mathrm{Grm}$. Krystallsoda in 3-4 Liter Wasser gegossen. Die zunächst

1) Ber. 25, 3545 .

2) Das. 25, 747.

3) Vgl. Wheelwright, Dissert., München 1893, S. 49.

4) Ann. Chem. 247, 217; s. auch Ber. 25, 3545 . 
224 Bamberger u.deGruy ter: Ueb.Formazylmethylketon. harzige, am Glasstab und der Gefässwandung festklebende Ausscheidung wird im Verlauf weniger Minuten bart und krystallinisch und kann nun bequem abgesaugt werden. Sie wird mit Eiswasser gewaschen und einmal aus siedendem Alkohol umkrystallisirt. Man erhält $95 \mathrm{Grm}$. chemisch reines Formazylmethylketon.

Molekulargewichtsbestimmung nach Raoult.

Phenol $=16,8$ Grm.

0,2636 Grm. gaben $0,46^{\circ}$ Depression. $M=252$.

0,5539 Grm. gaben $0,97^{\circ} \quad \# \quad M=251$.

$M$ ber. $=266$.

Bequemer, aber weniger ausgiebig ist die an anderer Stelle angegebene Methode der directen Kupplung der Acetessigsäure mit Diazobenzol. ${ }^{1}$ )

Umwandlung von Formazylmethylketon in Phenylaz of ormazyl ${ }^{2}$,

$\mathrm{CH}_{3}-\mathrm{CO}-\mathrm{C}\left\langle\aleph_{\mathrm{N}_{2} \mathrm{HC}_{6} \mathrm{H}_{5}}^{\mathrm{N}_{2} \mathrm{C}_{6} \mathrm{H}_{5}}+\mathrm{C}_{6} \mathrm{H}_{5} \mathrm{~N}_{2} \mathrm{OH}=\mathrm{CH}_{3} \mathrm{COOH}+\mathrm{C}_{6} \mathrm{H}_{6} \mathrm{~N}_{2}-\mathrm{C}\left\langle\mathrm{N}_{2} \mathrm{HC}_{6} \mathrm{H}_{5} \mathrm{~N}_{6} \mathrm{H}_{5}\right.\right.$

Das aus 1,74 Grm. Anilin in schwefelsaurer Lösung hergestellte Diazoniumsalz wurde in $160 \mathrm{Grm} .10$ procent. Kalilauge eingetragen und dann unter lebhaftem Umrühren bei $0^{0}$ der gut gekühlten Lösung von 5 Grm. Formazylmethylketon in einem Liter absoluten Alkohol hinzugefügt. Auf Zusatz von viel Wasser fällt ein braunrother Niederschlag aus (4 Grm.), welchem durch öfters wiederholte fractionirte Krystallisation aus kochendem Alkohol nicht mehr als 0,6 Grm. reines Phenylazoformazyl - schwarzrothe atlasglänzende Blättchen - entzogen werden konnten. Es wurde durch den Schmelzp. von $162^{\circ}$ und die charakteristische grünblaue Farbe der concentrirten schwefelsauren Lösung identificirt. Die Mutterlaugen ergaben 3,1 Grm. unverändertes Formazylmethylketon.

Zum Nachweis der Essigsäure engte man das alkoholischwässrige Filtrat ein, um es dann nach dem Uebersäuern mit Schwefelsäure der Dampfdestillation zu unterwerfen. Das Condensat wurde mit reinstem Natron neutralisirt, zur Trockne gebracht und im Rückstand das Natriumacetat mit aller Schärfe nachgewiesen (Bildung von Essigäther, Kakodyloxyd, Röthung

1) Ber. 25, 3545. $\quad$ 2) Das. S. 3539. 
Bamberger u.deGruy ter: Ueb.Formazylmethylketon. 225 mit Ferrichlorid). Ein Theil desselben wurde in das Silbersalk verwandelt und dieses, nachdem es aus kochendem $W$ asser umkrystallisirt war, analysirt.

0,1912 Grm. Substanz gaben 0,1237 Grm. Ag.

Berechnet für $\mathrm{CH}_{3} \mathrm{COOAg}$ : Gefunden:

$$
\mathrm{Ag}=64,67 \quad 64,69 \% \text {. }
$$

Metallsalze des Formazylmethylketons. ${ }^{1}$ )

Das Natriumsalz scheidet sich in hochrothen Prismen aus, wenn man der heissen, absolut alkoholischen Lösung von Formazylmethylketon die berechnete Menge Natriumäthylat hinzufügt und erkalten lässt. Durch Wasser wird es hydrolytisch zerlegt. Die lufttrockne Substanz enthält $1 \mathrm{Mol}$. Alkohol.

$0,6508 \mathrm{Grm}$. Substanz, bei $100^{\circ}$ getrocknet, verloren 0,0879 Grm.

0,206 Grm. Substanz gaben 0,0509 Grm. $\mathrm{Na}_{2} \mathrm{SO}_{4}$.

0,1221 Grm. Substanz gaben $21,7 \mathrm{Ccm}$. $\mathrm{N}$ bei $10^{\circ}$ und $699 \mathrm{Mm}$. B.

Ber. für $\mathrm{CH}_{3} . \mathrm{CO}-\mathrm{C} \bigcup_{\mathrm{N}_{2} \mathrm{NaC}_{6} \mathrm{H}_{5}}^{\mathrm{N}_{2} \mathrm{C}_{6} \mathrm{H}_{5}}+\mathrm{C}_{2} \mathrm{H}_{5} \mathrm{OH}: \quad$ Gefunden:

$$
\begin{aligned}
& \mathrm{C}_{2} \mathrm{H}_{6} \mathrm{O}=13,77 \quad 13,50 \% \\
& \mathrm{Na}=7,98 \quad 8,00 \% \\
& \mathrm{~N}=19,44 \quad 19,55 \ldots
\end{aligned}
$$

Das in gleicher Weise herstellbare Kaliumsalz hat ähnliche Eigenschaften.

Das Silbersalz fällt als schwarzes Pulver mit grünem Metallschimmer aus, wenn man dem Gemisch alkoholischer Lösungen von Formazylmethylketon $(0,3 \mathrm{Grm}$.) und Silbernitrat $(0,192 \mathrm{Grm}$.) tropfenweis ganz verdünntes Ammoniak hinzufügt. Es wurde mit Alkohol, Wasser und Aether gewaschen und im Vacuum zur Gewichtsconstanz gebracht.

I. $0,1795 \mathrm{Grm}$. Substanz gaben 0,052 Grm. Ag.

II. 0,1464 Grm. Substanz gaben $19,4 \mathrm{Cem}$. $\mathrm{N}$ bei $7^{\circ}$ u. $724 \mathrm{Mm}$. B.

Berechnet für $\mathrm{C}_{15} \mathrm{H}_{13} \mathrm{~N}_{4} \mathrm{Ag}$ :

$$
\begin{array}{ll}
\mathrm{Ag}=28,95 & 28,96 \% \\
\mathrm{~N}=15,02 & 15,38 \%
\end{array}
$$

Gefunden:

Acetylformazylmethylketon

haben wir nur einmal erbalten; die Acetylirung ist in diesem Fall (wie sehr häufig bei Formazylkörpern) eine äusserst subtile

1) Ber. 25, 3545.

Journal f. prakt. Chemie [2] Bd. 64. 
226 Bamberger u.deGruyter: Ueb.Formazylmethylketon.

Operation. 0,25 Grm. des Ketons wurde in $1 \mathrm{Grm}$. Essigsäureanhydrid gelöst, mit $0,15 \mathrm{Grm}$. Chlorzink versetzt und die Mischung wenige Secunden in kochendes Wasser getaucht. Sie ist erst roth, dann blau und wird in diesem Moment auf Schnee gegossen, wobei das Acetylderivat neben unverändertem Ausgangsmaterial in gelben Flocken ausfällt; erwärmt man zu lange, so schlägt die blaue Farbe in Braun um und die Operation ist misslungen. Man trennt die gelben Partien mechanisch, trocknet sie auf Thon und krystallisirt sie aus siedendem Ligroïn um, das sie beim Erkalten in bernsteingelben, durchsichtigen Prismen abscheidet. $O b$ der ihnen eigene Schmelzpunkt von $102^{\circ}$ bei weiterem Umkrystallisiren steigt, konnten wir wegen Materialmangel nicht feststellen. Erwärmt man die gelben Prismen mit Kali, so kehrt die rothe Farbe des Formazylmethylketons zurück.

Die Einwirkung von Phenylhydrazin auf Formazylmethylketon ${ }^{1)}$

führt - je nach der Versuchsanordnung - zu vier verschiedenen Substanzen.

Das normale Reactionsprodukt

Formazylmethylketon-Phenylhydrazon,

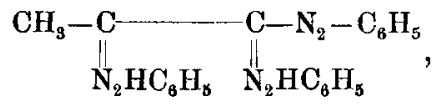

scheidet sich im Verlauf weniger Secunden aus, wenn die Lösung des Ketons (2,6 Grm.) in Eisessig (20 Ccm.) unter mässiger Kühlung mit essigsaurem Phenylhydrazin (1,1 Grm.) vermischt wird. Das nach etwa 3 Minuten als dicker Brei bronzeglänzender Flitterchen gefällte Hydrazon wird abgesaugt, in Chloroform gelöst und mit heissem Alkohol versetzt; nach dem Einengen krystallisiren beim Erkalten herrliche, diamant- oder pechglänzende schwarze Nadeln mit dunkel stahlblauem, metallischem Reflex. In Eisessig, Benzol, Alkohol, Aether schwer, leicht in Chloroform mit dunkelrother, fast schwarzer Farbe löslich. Schmelzpunkt $165^{\circ}$. Concentrirte Schwefelsäure nimmt die Krystalle mit tiefblauer Farbe auf.

1) Ber. 25, 3542 . 
Bamberger u.deGruyter: Ueb.Formazylmethylketon. 227

I. $0,1800 \mathrm{Grm}$. Subst. gaben $0,4691 \mathrm{Grm} . \mathrm{CO}_{2}$ u. $0,0948 \mathrm{Grm} . \mathrm{H}_{2} \mathrm{O}$.

II. $0,1254 \mathrm{Grm}$. Subst. gaben $27,5 \mathrm{Cm}$. $\mathrm{N}$ bei $19^{0}$ und $718 \mathrm{Mm}$. B.

Berechnet für $\mathrm{C}_{21} \mathrm{H}_{20} \mathrm{~N}_{8}$ :

$$
\begin{array}{lc}
\mathbf{C}=70,8 & \mathbf{7 1 , 0 7 \%} \\
\mathbf{H}=5,6 & \mathbf{5 , 8 5} \% \\
\mathbf{N}=23,6 & 23,7 \% .
\end{array}
$$

1-Phenyl-3-Phenylazo-4-Methylosotriazol,<smiles></smiles>

Verweilt das eben besprochene, zu einer undurchsichtigen Flüssigkeit geschmolzene Hydrazon einige Zeit in dem auf $170^{\circ}-180^{\circ}$ erbitzten Bade, so hellt sich die Schmelze allmählich auf, wird schliesslich bordeauxfarbig und erstarrt zu gelbrothen, sich gegen $110^{\circ}$ vertlüssigenden Krystallen.

Eine Wiederholung des Versuchs in grösserem Maassstab zeigte, dass es sich um einen unter Anilinaustritt erfolgenden Ringschluss handelt.

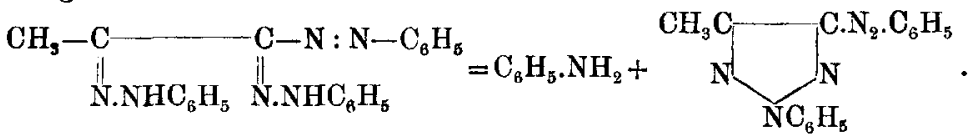

Das Anilin kann der erkalteten Schmelze durch einen Dampfstrom entzogen werden; der Triazolfarbstoff bleibt zurück.

Viel einfacher und eleganter lässt sich der Process verwirklichen, indem man die schwarzrothe, eisessigsaure Lösung des Formazylmethylketon-Phenylhydrazous - oder auch direct die Lösung der Componenten - einige Minuten lang (bei geringen Mengen wenige Secunden) im Kochen erhält; die zu Bordeauxroth aufgehellte Flüssigkeit scheidet auf vorsichtigen Wasserzusatz statt der blauschwarzen Nadeln des Osazons feine, mehrere Centimeter lange, goldgelbe Prismen von prächtigem Seidenglanz ab - chemisch reines Phenylazomethylosotriazol - und in der Lösung hinterbleibt Anilinacetat.

Der Farbstoff schmilat bei $122^{\circ}$ und wird von kochendem Alkohol leicht, schwierig ron kaltem aufgenommen; Wasser löst auch bei Siedetemperatur nur spurenweis, Ligroïn ziemlich leicht, Eisessig leicht, Aether und Chloroform spielend. Conccntrirte Mineralsäuren - am besten Schwefelsäure - 
228 Bambergeru.deGruy ter: Ueb.Formazylmethylketon.

lösen dic Krystalle mit dunkelorangerother Farbe und sctzen sie auf Wasserzusatz wieder ab.

I. $0,1114 \mathrm{Grm}$. Subst. gaben $0,2813 \mathrm{Grm} . \mathrm{CO}_{2}$ u. $0,0531 \mathrm{Grm} . \mathrm{H}_{2} \mathrm{O}$.

II. $0,1137 \mathrm{Grm}$. Subst. gaben $28,2 \mathrm{Cem}$. $\mathrm{N}$ bei $19^{\circ}$ und $718 \mathrm{Mm}$. B.

Berechnet für $\mathrm{C}_{15} \mathrm{H}_{13} \mathrm{~N}_{5}$ :

Gefunden:

$$
\begin{array}{lc}
\mathrm{C}=68,48 & 68,80 \% \\
\mathrm{H}=4,94 & 5,30 \% \\
\mathrm{~N}=26,6 & 26,9 \% .
\end{array}
$$

1-Phenyl-3-Amido-4-Methylosotriazol ${ }^{1}$ ),<smiles>CC1NN(C)C1=N</smiles>

entsteht bei der Reduction des Azofarbstoffs mit Zinnchlorür neben Anilin.

8 Grm. mit concentrirter Salzsäure verriebenes Phenylazomethylphenylosotriazol werden mit einer Lösung von Zinnchlorür in der nämlichen Säure vermischt. Die sich fast momentan entfärbende Flüssigkeit setzt einen hellen, in Wasser leicht löslichen Niederschlag ab. Man alkalisirt, entfernt das entstandene Anilin mit Wasserdampf und entzieht dem Rückstand das Aminotriazol mit Aether. Es hinterbleibt nach Entfernung des letzteren als gelbbraunes Oel, das mit kochendem Petroläther bis zur Erschöpfung extrahirt wird. Dunstet man das Lösungsmittel im trocknen Luftstrom ab, so krystallisirt die Base in zu Knollen vereinigten, gelblich weissen Nadeln, welche nochmals aus Petroläther umkrystallisirt rein weiss sind und den constanten Schmelzp. $83,5^{\circ}$ zeigen. Ausbeute an ganz reiner Base $=2 \mathrm{Grm}$. Leicht löslich in Alkohol, Benzol, Chloroform, Ligroïn, kaum in Wasser.

I. $0,1554 \mathrm{Grm}$. Subst. gaben $0,3526 \mathrm{Grm} . \mathrm{CO}_{2}$ u. $0,0841 \mathrm{Grm} . \mathrm{H}_{2} \mathrm{O}$.

II. $0,0771 \mathrm{Grm}$. Subst. gaben $22 \mathrm{Ccm}$. N bei $15^{\circ}$ und $730 \mathrm{Mm}$. B.

Berechnet für $\mathrm{C}_{8} \mathrm{H}_{10} \mathrm{~N}_{4}$ :

$$
\begin{array}{lr}
\mathrm{C}=26,06 & 61,87 \% \\
\mathrm{H}=5,74 & 6,01 \% \\
\mathrm{~N}=32,18 & 32,01 \%
\end{array}
$$

Gefunden:

Mineralsäuren nehmen die Base leicht auf und setzen sie auf Zusatz von Alkalien in weissen Nadeln wieder ab.

1) Vgl. Jagersbacher, Ber. 28, 1286. Weitere Bildungsweisen das. S. 1285 u. $26,2785$. 
Bamberger u.de(truyter: Ueb.Formazylmethylketon. 229

Ferrichlorid oder Bichromat färbt die sauren Lösungen roth. braun.

Phenyl - Amido - Methylosotriazolyl - Phenylharnstoff,<smiles>CCCCCCCCNC(=O)NC1CNNC1C</smiles>

fällt nach dem Mischen ätherischer Lösungen der Triazolbase und des Phenylcyanats sehr schnell als Krystallbrei aus.

Aus erkaltendem Alkohol feine weisse Nadeln vom Schmelzp. $240^{\circ}$. Alkohol, Benzol, Chloroform lösen bei gewöhnlicher Temperatur schwer, Eisessig leicht, Aether und Ligroin sehr wenig.

0,0888 Grm. Subst. gaben $19,45 \mathrm{Ccm}$. $\mathrm{N}$ bei $19^{\circ}$ und $727 \mathrm{Mm}$. B.

Berechnet für $\mathrm{C}_{16} \mathrm{H}_{15} \mathrm{~N}_{8} \mathrm{O}$ :

$$
\mathrm{N}=23,89
$$

Gefunden:

$24,11 \%$.

Phenyl-Amido-Methylosotriazolyl-Phenylthioharnstoff,

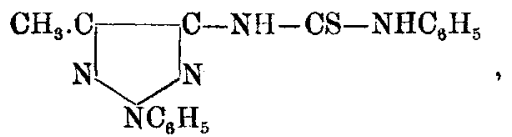

krystallisirt in weissen, glasglänzenden Nadeln aus, wenn man die concentrirt alkoholische Lösung des amidirten Osotriazols mit der berechneten Menge Phenylsenföl einige Minuten lang zum Sieden erhitzt und dann abkühlt; der grösste Theil scheidet sich aus der Mutterlauge aus. Alkohol, Benzol, Aether lösen in der Kälte schwierig, Chloroform sehr leicht. Schmelzp. $195^{\circ}$. $0,0858 \mathrm{Grm}$. Subst. gaben $17,95 \mathrm{Ccm}$. N bei $19^{\circ}$ und $722 \mathrm{Mm}$. B.

Berechnet für $\mathrm{C}_{16} \mathrm{H}_{15} \mathrm{~N}_{5} \mathrm{~S}$ :

$$
\mathrm{N}=22,65
$$

Gefunden: $22,78 \%$.

1-Phenyl-3-Oxy-4-Methylosotriazol,<smiles>CCCCN1NC2CCCC21C(=O)O</smiles>

$1 \mathrm{Grm}$. Phenylamidomethylosotriazol wird in 8-10 Mol. Schwefelsäure $(1: 2)$ gelöst und bei $0^{\circ}$ mit $0,413 \mathrm{Grm}$. Natriumnitrit versetzt. Die eventuell zu filtrirende Diazoniumlösung, 
230 Bamberger u.deGruyter: Ueb.Formazylmethylketon.

welche mit Phenolaten intensiv kuppelt, wird nach dem Verdünnen mit Wasser zum Sieden erhitzt oder längere Zeit an einem warmen Orte stehen gelassen. Unter Stickstoffentwicklung fällt das Phenol in gelbbraunen, recht unreinen Flocken aus. Man reinigt es (nachdem man zuvor den Rest aus dem Filtrat mittels Aether gesammelt hat) durch Krystallisation aus Ligroïn. Ausbeute gering. Gelbliche Nädelchen vom Schmelzp. $140^{\circ}-142^{\circ}$. Aether, Benzol, Chloroform, Alkohol lösen schon kalt leicht, Ligrö̈n schwierig. Natronlauge nimmt die Krystalle unschwer auf, Säuren fällen sie wieder aus.

0,1250 Grm. Subst. gaben 27,1 Ccm. N bei $15^{\circ}$ und $732 \mathrm{Mm}$. B.

Berechnet fürr $\mathrm{C}_{9} \mathrm{H}_{9} \mathrm{~N}_{3} \mathrm{O}$ :

$\mathrm{N}=24,00$

Gefunden :

$24,38 \%$.

Wie oben erwähnt, verwandelt sich Formazylmethylketon unter der Linwirkung des Phenylhydrazins je nach den Versuchsbedingungen entweder in sein Osazon (Schmelzp. 165 ${ }^{\circ}$ ) oder aber in Phenylazomethylosotriazol (Schmelzp. 122 ${ }^{\circ}$ ). Ein wieder anderes Resultat ergiebt sich, wenn man jene zwei Körper ohne Verdünnungsmittel zu gleichen Theilen oder auch in äquimolekularen Mengen auf dem Wasserbad schwach anwärmt. Das Gemisch verflüssigt sich und reagirt plötzlich in so stürmischer Weise, dass Vorsicht erforderlich ist.

Der Kolbeninhalt erstarrt beim Erkalten zu einem gelbrothen Krystallbrei, welcher durch fractionirte Krystallisation aus heissem Benzol in seine Bestandtheile zerlegt werden kann. Der leichter lösliche erweist sich als das bald zu beschreibende Acetylamidrazon (Schmelzp. $183^{\circ}$ ), der andere als das Phenylhydrazon des letzteren (Schmelzp. 224\%). Das Phenylhydrazin. wirkt also unter diesen Umständen als Reductionsmittel auf das Formazylmethylketon:

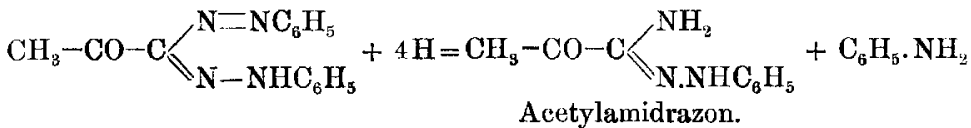

Das Acetylamidrazon wird dann durch den Ueberschuss des Phenylhydrazins theilweise in sein Osazon verwandelt. Nachdem das Wesen der Reaction erkannt war, hatte man 
Bamberger u.degrny ter: Ueb.Formaylmethylketon. 231

es einigermaassen in seiner Gewalt, die Bildung des einen oder des anderen der zwei Reactionsprodukte zu begünstigen. Frrwärmt man äquimolekulare Mengen von Formazylnethylketon und Phenylhydrazin auf dem Wasserbad - bei beginnender Reaction ist das Gefäss zu entfernen - und giesst sogleich nach beendeter Einwirkung das heisse Gemisch in gekühltes Benzol, so wird die Osazonbildung fast ganz vermieden und man erhält nahezu ausschliesslich das Acetylamidrazon. Wünscht man dagegen das Osazon desselben, so vermehrt man die Menge des Phenylhydrazins und setzt das Erwärmen etwas länger fort. Ueber die Eigenschaften beider Körper s. unten.

\section{Einwirkung von Salzsäure auf Formazylmethylketon. ${ }^{1}$ )}

$$
\alpha \text {-Phentriazyl-Methylketon, }
$$<smiles>COC(=O)C1NC2CCCCC2C1N</smiles>

Dieser Körper entsteht im Sinne der Gleichung

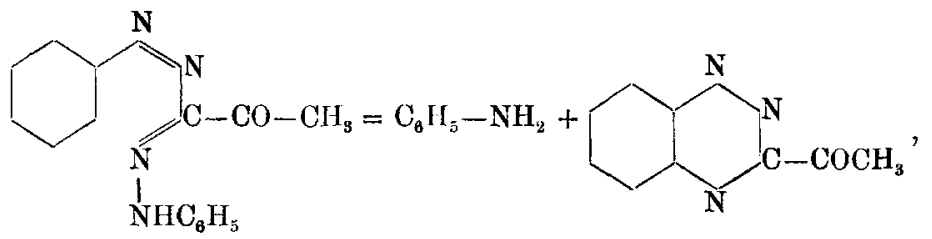

wenn man Formazylmethylketon (10 Grm.) in kleinen Portionen mit der 30-40 fachen Menge concentrirter Salzsäure unter fortwährendem Umschwenken über freier Flamme erhitzt. Die violette Farbe der Lösung verschwindet nach wenigen Minuten, um einer schmutzig grünbraunen Platz zu machen. Der schliesslich ganz klare Kolbeninhalt wird sofort in kaltes Wasser gegossen, wodurch ein Theil des Reactionsproduktes in wenig verharztem Zustand ausgefällt wird. Man filtrirt, nimmt den Rückstand in concentrirter Salzsäure auf, filtrirt wieder durch Asbest und lässt die Lösung in Wasser einfliessen; die von einer etwaigen Ausscheidung befreite Flüssigkeit wird, nachdem sie zuvor neutralisirt ist, gemeinsam mit dem früheren Filtrat sehr

\footnotetext{
1) Ber. 25, 3540.
} 
232 Bamberger u.deGruyter: Ueb.Formazylmethylketon.

oft - am besten unter Zusatz von Ammonsulfat - mit Chloroform ausgeschüttelt.

Krystallisirt man den Rückstand dieses Extractes aus kochendem Ligroïn um, so schiesst aus der vom Harz durch Filtration befreiten Lösung beim Erkalten das cyclische Keton in goldgelben, glitzernden, constant bei $121,5^{\circ}-122,5^{\circ}$ schmelzenden Nadeln an, welche von concentrirten Mineralsäuren gelöst werden und beim Verdünnen mit der richtig bemessenen Wassermenge wieder ausfallen. Kochendes Wasser nimmt sie ziemlich leicht, kaltes viel weniger auf. Alkohol, Benzol, Eisessig, Aether und besonders Chloroform lösen leicht, Ligroïn in der Kälte sehr schwer, kochend mässig. Ausbeute 1,75 Grm.

Der nämliche Körper entsteht auch, wenn man Formazylmethylketon mit Schwefelsäure (1 Gew.-Th. +1 Gew.-Th. Wasser) auf dem Wasserbad erwärmt. Man wird - beiläufig bemerkt die Darstellungsmethode erheblich verbessern können.

I. 0,1641 Grm. Subst. gaben 0,3755 Grm. $\mathrm{CO}_{2}$ u. $0,0596 \mathrm{Grm}$. $\mathrm{H}_{2} \mathrm{O}$.

II. $0,1490 \mathrm{Grm}$. Subst. gaben $32,6 \mathrm{Ccm}, \mathrm{N}$ bei $10^{\circ}$ u. $716 \mathrm{Mm}$. B.

Berechnet für $\mathrm{C}_{8} \mathrm{H}_{7} \mathrm{~N}_{3} \mathrm{O}$ :

$$
\begin{array}{lc}
\mathrm{C}=62,44 & 62,41 \% \\
\mathrm{H}=4,06 & 4,02 \% \\
\mathrm{~N}=24,28 & 24,66 \%
\end{array}
$$

Gefunden:

In der sauren, vom Keton durch Ausschütteln mit Chloroform befreiten Lösung befindet sich Anilin. Es wurde als solches und in Form von Acetanilid identificirt.

Erwärmt man das Acetylphentriazin auf dem Wasserbad mit einer Lösung von Zinnchlorür in concentrirter Salzsäure, so scheiden sich gelbbraune Krystalle $a b$, welche abgesaugt, mit Säure gewaschen und, nachdem sie mit Natron zersetzt waren, in Aether aufgenommen wurden. Der etwas dunkle Rückstand des Extracts liess sich durch Krystallisation aus Wasser unter Anwendung von Thierkohle leicht entfärben. Aus erkaltendem Wasser oder Benzol kleine, schief abgeschnittene, kalkspathähnliche Prismen vom Schmelzp. 165; schwer löslich in kaltem, viel leichter in siedendem Wasser, ziemlich schwer in Benzol, leichter in Alkohol und Aether, leicht in Chloroform, sehr schwer in Ligroĩn löslich. Das Reductionsprodukt wurde bisher nur in geringer Menge dargestellt und ist nicht eingehender untersucht worden. 
Phenylhydrazon des $\alpha$-Phentriazyl-Methylketons<smiles>CCCCCCCC(C)=NC1CNC2CCCCC2N1</smiles>

Erwärmt man die Lösung von $0,45 \mathrm{Grm}$. des Ketons in $6 \mathrm{Ccm}$. absolutem Alkohol nach Zugabe von 0,18 Grm. Phenylhydrazin wenige Augenblicke über freier Flamme, so scheidet sich nach kurzem Stehen das Hydrazon in fast berechneter Menge als azobenzolartig aussebender Niederschlag ab. Einmalige Krystallisation aus Alkohol genügt, um es analysenrein zu machen.

Atlasglänzende, flache, ziegelrothe Nadeln, welche - nachdem sie zuvor dunkler geworden und erweicht sind - bei $202^{\circ}$ schmelzen

Löslichkeit: Alkohol kalt sehr schwer; kochend leichter.

Aether schwer (hellgelb).

Ligroïn sehr schwer (hellgelb).

Aceton und Benzol heiss leicht (orangeroth), kalt viel schwerer.

Chloroform leicht.

Die Farbe der concentrirt schwefelsauren Lösung ist grün. braun und schlägt auf Zusatz von etwas Bichromat oder Eisenchloridlösung in Blau um.

I. 0,1607 Grm. Subst. gaben 0,4025 Grm. $\mathrm{CO}_{2}$ u. 0,0755 Grm. $\mathrm{H}_{2} \mathrm{O}$.

II. 0,1110 Grm. Subst. gaben $25,5 \mathrm{Ccm}$. $\mathrm{N}$ bei $5^{\circ}$ und $725 \mathrm{Mm}$. B.

Berechnet für $\mathrm{C}_{15} \mathrm{H}_{13} \mathrm{~N}_{5}$ :

$$
\begin{array}{lc}
\mathrm{C}=68,44 & \text { Gefunden : } \\
\mathrm{H}=4,94 & 68,30 \% \\
\mathrm{~N}=26,62 & 5,21 \% \\
& 26,80 \%
\end{array}
$$

Die

Einwirkung von Schwefelammonium auf Formazylmethylketon $^{1}$ )

entspricht der Gleichung:

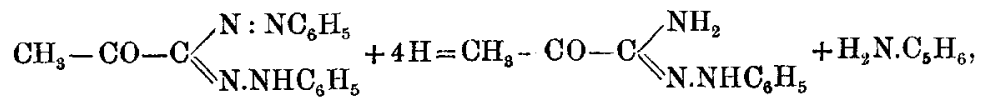

1) Ber. 25, 3541 u. 26, 2783. 
234 Bamberger u. deGruyter: Ueb.Formazylmethylketon.

fïbrt also zu einer Mischung von Auilin und

$$
\text { Acetylamidrazon, }
$$

Dass diese Base auch mittelst Phenylhydrazin erhalten werden kann, ist bereits oben bemerkt. Zu ihrer Darstellung verfährt man folgendermaassen:

Man leitet in die Suspension von $10 \mathrm{Grm}$. Formazylmethylketon in etwa $100 \mathrm{Grm}$. absolutem Alkohol bei $0^{n}$ trocknes Ammoniakgas bis zur Sättigung und darauf Schwefelwasserstoff ein, bis die Krystalle gelöst sind und die anfangs rothe Farbe der Flüssigkeit in Braungelb umgeschlagen ist; gelindes Erwärmen beschleunigt die Reduction erheblich. Die Lösung wird nun kurze Zeit gekocht, von Schwefel heiss abfiltrirt, fast zur Trockne eingedampft und mit Wasserdampf zur Vertreibung des Anilins behandelt. Das im Rückstand befindliche Acetylamidrazon ist mit verdünnter Salzsäure aufzunehmen und, nachdem es von beträchtlichen Mengen Schwefel durch Filtration befreit ist, mit Ammoniak zu fällen. Ausgewaschen und auf Thon getrocknet, beträgt es 6,5 Grm. (Schmelzp. 177\%); es ist, einmal aus kochendem Benzol umkrystallisirt, analysenrein.

Gelbe flache atlasglänzende Nadeln vom Schmelzp. 183", welche innerhalb des Lösungsmittels, aus dem sie sich ausscheiden, violett leuchten ${ }^{1}$ ); leicht in Mineralsäuren und Essigsäure löslich und durch Alkalien wieder fällbar. Kochendes Wasser nimmt die Base schwer auf und setzt sie beim Erkalten fast vollständig als haarfeine, flache, stark lichtbrechende, atlasglänzende Nadeln ab. Alkohol, Eisessig, Aether, Chloroform sind schon bei Zimmertemperatur gute Lösungsmittel. Benzol dagegen nimmt in der Kälte so wenig auf, dass die kochend gesättigte Lösung beim Abkühlen etwa fünf Sechstel der Substanzmenge wieder ausscheidet. Heisses Ligroin löst ziemlich wenig, kaltes fast gar nicht.

I. 0,1986 Grm. Subst. gaben $0,4435 \mathrm{Grm} . \mathrm{CO}_{2}$ u. 0,1137 Grm. $\mathrm{H}_{2} \mathrm{O}$.

II. $0,3183 \mathrm{Grm}$. Subst. gaben $0,7117 \mathrm{Grm} . \mathrm{CO}_{2}$ u. $0,1765 \mathrm{Grm} . \mathrm{H}_{2} \mathrm{O}$. III. $0,15,56$ Grm. Subst. gaben $33 \mathrm{Cem}$. $\mathrm{N}$ bei $11^{\circ}$ und $720 \mathrm{Mm}$. 13 .

1) Besonders bei Verwendung von Ligroïn. 
Bamberger u.deGruy ter: Ueb.Formazylmethylketon. 235

$$
\begin{array}{crc}
\text { Berechnet für } \mathrm{C}_{9} \mathrm{H}_{11} \mathrm{~N}_{3} \mathrm{O}: & \multicolumn{2}{c}{\text { Gefunden: }} \\
\mathrm{C}=61,02 & 60,88 & 60,99 \% \\
\mathrm{H}=6,21 & 6,35 & 6,16 \\
\mathrm{~N}=23,73 & 23,87 & -. \\
\text { Acetyl-Acetylamidrazon } & \\
& \\
\mathrm{CH}_{3}-\mathrm{CO}-\mathrm{C} /{ }_{\mathrm{N} . \mathrm{NHC}_{6} \mathrm{H}_{5}}^{\mathrm{NH}\left(\mathrm{COCH}_{3}\right)},
\end{array}
$$

entsteht nur bei Innehaltung bestimmter Versuchsbedingungen, da es ungemein leicht in das gleich zu beschreibende Triazolsystem übergeht.

3 Grm. Acetylamidrazon werden unter beständigem Verreiben bei $40^{\circ}-45^{\circ}$ in $30 \mathrm{Grm}$. Essigsäureanhydrid gelöst. Aus der dunkelbraunen Flüssigkeit scheiden sich nach $1 \frac{1}{2}$ bis 2 Stunden ruhigen Stehens an den Wandungen des Gefässes gelbe Nädelchen $a b$, welche im Verlauf weiterer vier Stunden die ganze Lösung durchsetzen. Sie werden abgesaugt, mit wenig Acetanhydrid gewaschen, auf Thon getrocknet $(0,7 \mathrm{Grm}$.$) , in wenig heissem Benzol gelöst und mit der vier-$ fachen Menge erwärmten Ligroïns versetzt. Beim Erkalten krystallisiren gelbweisse, sternförmig gruppirte, atlasglänzende Nadeln, welche nach zwei- bis dreimal wiederholter Reinigung constant bei $143^{\circ}$ schmelzen. Unlöslich in verdünnten Säuren. Fast unlöslich in Wasser und Ligroïn, spielend in Chloroform, ziemlich leicht in Aether löslich.

0,1169 Grm. Subst. gaben 0,2591 Grm. $\mathrm{CO}_{2}$ und 0,0645 Grm. $\mathrm{H}_{2} \mathrm{O}$. $0,0965 \mathrm{Grm}$. Subst. gaben $16,9 \mathrm{Ccm}$. $\mathrm{N}$ bei $10^{\circ}$ und $703 \mathrm{Mm}$. B.

Bercchnet für $\mathrm{C}_{11} \mathrm{H}_{18} \mathrm{~N}_{3} \mathrm{O}_{3}$ :

$$
\begin{aligned}
& \mathrm{C}=60,27 \\
& \mathrm{H}=5,93 \\
& \mathrm{~N}=19,18
\end{aligned}
$$

$$
\begin{gathered}
\text { Gefunden: } \\
60,46 \% \\
6,12 \% \\
19,24 \%
\end{gathered}
$$

Die Mutterlauge des Rohprodukts verwendet man zur Darstellung der folgenden Verbindung $(2,5 \mathrm{Grm}$.$) , welche immer$ entsteht, wenn man bei der Acetylirung längere Zeit oder auf höhere Temperatur erhitzt:

1) Den von Herrn de Gruyter dargestellten Harnstoff (s. seine Dissert. S. 57), welcher auch in den Berichten der D. chem. Gresellschaft erwähnt ist $(\mathbf{2 6}, 2784)$, haben wir nicht mit aufgenommen, da er vielleicht nur unverändertes Acetylamidrazon daratellt. 
236 Bamberger u.deGruyter: Uob. Formazylmethylketon<smiles>CCNN=C(NC(=O)C[PH2+])C(C)=O</smiles>

1-Phenyl-2-Methyl-4-Acetyltriazol,<smiles>CC(=O)C1NC2(C)NC12</smiles>

leicht erhältlich aus der eben erwähnten Essigsäureanhydridlauge, indem man dieselbe mit Wasser versetzt und zur Trockne eindampft. Am einfachsten stellt man es durch mehrstündiges Kochen einer Lösung des Acetylamjdrazons in Essigsäureanhydrid dar. Man entfernt den Ueberschuss des letzteren durch wiederholtes Eindampfen mit Wasser und krystallisirt das zurückbleibende, beim Erkalten erstarrende Oel aus kochendem Wasser oder Ligrö̈n (Thierkohle) um. Ausbeute quantitativ.

Centimeterlange, glasglänzende, weisse, büschelförmig gruppirte Nadeln vom Schmelzp. $88^{\circ}-89^{\circ}$. Sehr leicht in heissem, ziemlich in kaltem Wasser, spielend in Eisessig und besonders in Chloroform, leicht in Aether, mässig in kochendem Ligroïn und recht schwer in kaltem löslich. Mineralsäuren nehmen sie leicht auf und scheiden sie auf Zusatz von Alkalien wieder ab.

$0,1499 \mathrm{Grm}$. Subst. gaben $0,3588 \mathrm{Grm} . \mathrm{CO}_{2}$ und 0,073 Grm. $\mathrm{H}_{2} \mathrm{O}$. $0,2159 \mathrm{Grm}$. Subst. gaben 0,520 Grm. $\mathrm{CO}_{2}$ und 0,1044 Grm. $\mathrm{H}_{2} \mathrm{O}$.

$0,1710 \mathrm{Grm}$. Subst. gaben $32 \mathrm{Ccm}$. $\mathrm{N}$ bei $14^{\circ}$ und $720 \mathrm{Mm}$. B.

0,1496 Grm. Subst. gaben $28 \mathrm{Ccm}$. N bei $10^{\circ}$ und $715 \mathrm{Mm}$. B.

Berechnet für $\mathrm{C}_{11} \mathrm{H}_{11} \mathrm{~N}_{3} \mathrm{O}$ :

$$
\begin{aligned}
& \mathrm{C}=65,67 \\
& \mathbf{H}=\mathbf{5 , 4 7} \\
& \mathbf{N}=20,89
\end{aligned}
$$

Gefunden:

$\begin{array}{rrr}65,28 & 65,7 & \% \\ 5,41 & 5,4 & " \\ 20,91 & 21,01 & \% \text {. }\end{array}$

Natriumbisulfitverbindung des Phenylmethylacetyltriazols,<smiles>CC(C)C1NC1C(C)(O)[S+](=O)[O-]</smiles>

0,5 Grm. der Triazolbase werden mit einem kleinen Ueberschuss 40 procent. Bisulfitlösung erwärmt. Beim Erkalten 
Bambergeru.deGruyter: Ueb.Formazylmethylketon. 237

scheiden sich glänzend weisse Krystalle aus, welche nach 15 Minuten abgesaugt, mit wenig Wasser (das leicht löst), dann mit Alkohol und Aether gewaschen werden.

0,2007 Grm. Substanz gaben 0,0485 Grm. $\mathrm{Na}_{2} \mathrm{SO}_{4}$.

Ber. für $\mathrm{C}_{11} \mathrm{H}_{12} \mathrm{~N}_{3} \mathrm{O}_{4} \mathrm{SNa}$ :

Gefunden:

$$
\mathrm{Na}=7,54
$$

$7,82 \%$.

Oxim des Phenylmethylacetyltriazols,<smiles>CCCN1C2NC(N2)C1C</smiles>

Die concentrirt alkoholische Lösung von 0,95 Grm. Phenylmethylacetyltriazol wird mit einer Lösung von $0,35 \mathrm{Grm}$. Hydroxylaminchlorhydrat $(1 \mathrm{Mol}$.$) und 0,8 \mathrm{Grm}$. Soda $(1,5 \mathrm{Mol}$.) in $15 \mathrm{Ccm}$. Wasser versetzt; ein etwa auftretender Niederschlag verschwindet bei Zusatz von etwas mehr Wasser. Einmaliges kurzes Aufkochen genügt zur Oximirung. Nach zweistündigem Stehen krystallisirt das Oxim in weissen, federartig gruppirten Nadeln, welche aus Alkohol in Form grosser rbombischer Tafeln krystallisiren. Schmelzp. $211^{\circ}-212^{\circ}$. Leicht löslich in verdünnten Säuren und auf Zusatz von Ammoniak wieder ausfallend.

0,1611 Grm. Subst. gaben $0,3607 \mathrm{Grm} . \mathrm{CO}_{2}$ und 0,0857 Grm. $\mathrm{H}_{2} \mathrm{O}$. $0,077 \mathrm{Grm}$. Subst. gaben $17,8 \mathrm{Ccm}$. $\mathrm{N}$ bei $11^{\circ}$ und $720 \mathrm{Mm}$. B.

Berechnet für $\mathrm{C}_{11} \mathrm{H}_{12} \mathrm{~N}_{4} \mathrm{O}$ :

$$
\begin{array}{lr}
\mathrm{C}=61,11 & 61,06 \% \\
\mathrm{H}=5,55 & 5,91 \% \\
\mathrm{~N}=25,92 & 26,06 \%
\end{array}
$$

Gefunden:

Phenylhydrazon des Phenylmethylacetyltriazols,<smiles>CCCN1C(C)C2CN(CC)CC21</smiles>

Vermischt man die Lösungen von $1 \mathrm{Grm}$. Phenylmethylacetyltriazol und 0,54 Grm. Phenylhydrazin in verdünter Essigsäure, so erfüllt sich die Flüssigkeit alsbald mit Oeltröpfchen, welche bei anhaltendem Reiben erstarren $(1,3 \mathrm{Grm}$.$) .$ Aus erkaltendem Alkohol oder Benzol scheidet sich das Hydrazon in diamantglänzenden, sternförmig gruppirten, durch Thier- 
238 Bamberger u.deGruyter: Ueb.Formazylmethylketon.

kohle leicht za entfärbenden Nadeln ab. Dieselben wurden erst aus Alkohol, dann aus Benzol und zum Schluss wieder aus Alkohol umkrystallisirt, ohne dass ein ganz scharfer Schmelzpunkt erzielt werden konnte. ${ }^{1}$ ) Heizt man allmählich an, so erweichen die Krystalle gegen $130^{\circ}$ und verflüssigen sich bei $133,5^{\circ}$ (corr.) zu einem trüben Syrup; taucht man dagegen die nämliche Probe in ein auf $110^{\circ}-112^{\circ}$ vorgeheiztes Bad, so schmilzt sie sofort unter Gasentwicklung. Auf diese eigenthümlichen Verhältnisse näher einzugehen, fehlte es uns an Zeit. Vielleicht liegt ein Gemisch von Raumisomeren vor. Analyse der zwischen Fliesspapier gepressten und kurze Zeit (15-20 Minuten) im Vacuum über Schwefelsäure getrockneten Krystalle:

0,1785 Grm. Subst. gaben 0,4606 Grm. $\mathrm{CO}_{2}$ und $0,0963 \mathrm{Grm}$. $\mathrm{II}_{2} \mathrm{O}$. Berechnet für $\mathrm{C}_{17} \mathrm{H}_{17} \mathrm{~N}_{5}$ :

$$
\begin{aligned}
& \mathrm{C}=70,1 \\
& \mathbf{H}=5,84 \\
& 70,38 \% \\
& 5,99 \text {,. }
\end{aligned}
$$

Gefunden:

1-Phenyl-2-Methyl-Triazol-4-Carbonsäure ${ }^{2}$ ),<smiles>CC1(C)NC(C(=O)O)N1c1ccccc1</smiles>

entsteht durch Oxydation des 1-Phenyl-2-Methyl-4-Acetyltriazols mit Kaliumpermanganat bei Gegenwart von Soda. Glasglänzende Nadeln vom Schmelzp. $177^{\circ}-177,5^{\circ}$, identisch mit der von Bladin aus Cyanamidrazon erhaltenen Säure.

Silbersalz, $\mathrm{C}_{10} \mathrm{H}_{8} \mathrm{~N}_{3} \mathrm{O}_{2} \mathrm{Ag}+1,5 \mathrm{H}_{2} \mathrm{O}$.

Kupfersalz, $\left(\mathrm{C}_{10} \mathrm{H}_{8} \mathrm{~N}_{3} \mathrm{O}_{2}\right)_{2} \mathrm{Cu}+1,5 \mathrm{H}_{2} \mathrm{O}$.

Nitroderivat. Tafelförmige Prismen, Schmelzp. $185^{\circ},=$<smiles>CC(C)NC(NC(=O)[O-])C(=O)O</smiles>

1) Die Angabe de Gruyter's über den Gehalt der Krystalle an Essigsäure, welche sich in der Dissertation des Genannten (S.62) findet und in unsere vorläufige Mittheilung (Ber. 26, 2786) übernommen wurde, habe ich nicht bestätigt gefunden. Zu näherer Untersuchung der eigenartigen Schmelzpunktserscheinungen gebrach es mir an Material. Bamberger.

2) Dieses und die nachfolgenden Triazolderivate sind nur kurz erwähnt, da sie ausführlich schon von uns in den Ber. 26, 2385 behandelt sind. 
Bamberger u.deGruy ter: Ueb.Formazylmethylketon. 239

$$
\begin{aligned}
& \text { 1-Phenyl-2-Methyltriazol'), } \\
& \text { C.CH, }
\end{aligned}
$$

entstcht beim Erhitzen der 1-Phenyl-2-Methyl-Triazol-4-Carbonsäure oder auch (weniger gut) ihres mit Thonkachelpulver gemischten Silbersalzes. Lange, farblose Prismen vom Schmelzp. $191^{\circ}$, von Bladin als Oel erhalten.

$$
\text { 1. Phenyltriazol-4. Carbonsäure, }
$$

Sowohl durch Oxydation der 1-Phenyl-2-Methyltriazol4-Carbonsäure als auch durch directe Oxydation des oben beschriebenen 1-Phenyl-2-Methyl-4-Acetyltriazols erhälttich, wenn man die Krystallsoda (s. oben) durch Kaliumhydroxyd ersetzt. Weisse Nadeln vom Schmelzp. $185^{\circ}$, identisch mit der schon von Bladin dargestellten Säure.

Der aus dem Silbersalz mit Jodmethyl dargestellte Methyläther bildet wasserhelle Tafeln vom Schmelzp. 116,5 - ganz wie es Bladin angegeben hat.

Phenylhydrazon des Acetylamidrazons ${ }^{2}$ ),

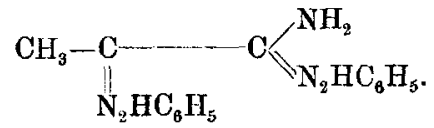

$3 \mathrm{Grm}$. Acetylamidrazon wurden mit $18 \mathrm{Grm}$. unverdünntem Phenylhydrazin übergossen und unter beständigem Umschwenken etwa 10 Minuten lang im Oelbad auf $115^{\circ}-120^{\circ}$ erhitzt; bei etwa $110^{\circ}$ löst sich das Amidrazon auf und das bei der Reaction erzeugte Wasser verdampft. Sobald sich an der Glaswandung Krystalle abzusetzen beginnen, giesst man die noch heisse Lösung in gut gekühltes Benzol und spült mit letzterem

1) Von Herm de Gruyter in München dargestellt, als ich bereits nach Zürich übergesiedelt war.

Bamberger.

2) Ber. 26, 2785. Ueber ein Isomeres vgl. Jagersbacher, das. 28,1284 . 
240 Bamberger u.deGruyter: Ueb.Formazylmethylketon.

nach. Das Osazon scheidet sich beim Erkalten in grosser Menge als weissgelbes, zu Boden sinkendes Pulver aus (3,4 Grm. Schmelzp. $221^{\circ}$ ).

Man krystallisirt es aus kochendem Benzol um (auf 5 Grm. Substanz ca. 200 Grm.) und erhält es als weisses Krystallpulver vom Schmelzp. $224^{\circ}$; aus Ligroïn kleine wasserhelle Nädelchen. Ueberzieht sich an der Luft allmählich mit einer rothvioletten Oxydationsschicht. In Aether, Chloroform und Aceton leicht, in Ligroïn - selbst kochendem - wenig löslich. Concentrirte Schwefelsäure nimmt es mit intensiv blauvioletter Farbe auf. Alkalien färben die Base oder ibre alkoholische Lösung roth.

0,1554 Grm. Subst. gaben 0,3835 Grm. $\mathrm{CO}_{2}$ und 0,0913 Grm. $\mathrm{H}_{2} \mathrm{O}$. 0,1398 Grm. Subst. gaben 0,3466 Grm. $\mathrm{CO}_{2}$ und $0,0827 \mathrm{Grm}$. $\mathrm{H}_{2} \mathrm{O}$. 0,1254 Grm. Subst. gaben 29 Cerr. $N$ bei $6^{0}$ und $711 \mathrm{Mm}$. B.

Berechnet für $\mathrm{C}_{15} \mathrm{H}_{17} \mathrm{~N}_{5}$ :

Gefunden:

$$
\begin{array}{lrr}
\mathbf{C}=67,41 & 67,31 & 67,59 \% \\
\mathbf{H}=6,37 & 6,52 & 6,57, \\
\mathbf{N}=26,22 & 26,30 & -
\end{array}
$$

Die Farblosigkeit der Substanz hat in uns Bedenken an ihrer Osazonnatur erregt; indess wüssten wir in Anbetracht ihrer Entstehungsweise und ihrer procentualen Zusammensetzung keine andere als die in obiger Formel ausgedrückte Deutung zu geben. ${ }^{1}$ )

Die farblose Base bildet mit Mineralsäuren stark gelb gefärbte, prächtig krystallisirende, in heissem Wasser leicht, in kaltem (überschüssige Säure enthaltendem) schwer lösliche Salze. Das Chlorhydrat wird aus der wässrigen Lösung schon durch wenige Tropfen verdünnter Salzsäure abgeschieden. Zum Umkrystallisiren der Salze empfiehlt sich absoluter Alkohol. Das Sulfat schmilzt bei $211^{\circ}$, das salzsaure Salz bei $114^{\circ}-115^{\circ}$, indem es Chlorwasserstoff verliert; nach dem Abkühlen nochmals erhitzt, zeigt es annähernd den Schmelzpunkt der freien Base $224^{\circ}$.

1) Besonders, da der Körper in das zuvor beschriebene PhenylA mido-Methylosotriazol

üb erführbar ist.

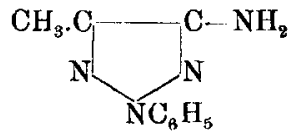


Dass sich das Osazon des Acetylamidrazons auch unter den Produkten der Einwirkung von Phenylhydrazin auf Formazylmethylketon befindet, ist bereits oben erwähnt worden.

Acetylamidrazon und verdünnte Schwefelsäure.

$3 \mathrm{Grm}$. Acetylamidrazon wurden so lange mit kochender verdünnter Schwefelsäure (1 Gew.-Th. : 6 Gew.-Th. Wasser) in Berührung gelassen, bis sich die ersten Harzspuren zeigten; die alsdann heiss filtrirte Lösung setzte beim Erkalten ein bald erstarrendes Oel ab. Indem das Filtrat noch viermal in gleicher Weise behandelt wurde, liessen sich im Ganzen 2 Grm. des nämlichen (gelbgefärbten) Körpers gewinnen, welcher sich als das eben beschriebene Sulfat des Acetylamidrazon - Osazons zu erkennen gab; dieses selbst fiel unter gleichzeitiger Ammoniakentwicklung aus, als das letzte schwefelsaure Filtrat mit Soda alkalisirt wurde.

Dass die gelben Krystalle in der That das Osazonsulfat sind, ergab sich, als ihre Lösung in kochendem, schwefelsäurehaltigem Wasser mit Natriumcarbonat versetzt wurde: es fiel das weisse Osazon in reinem Zustand (Schmelzp. 224 ${ }^{\circ}$ ) nieder.

$0,135 \mathrm{Grm}$. Subst. gaben $31,2 \mathrm{Ccm}$. $\mathrm{N}$ bei $12^{\circ}$ und $728 \mathrm{Mm}$. B.

Berechnet für $\mathrm{C}_{15} \mathrm{H}_{17} \mathrm{~N}_{5}$ : $\mathrm{N}=26,22$

Gefunden: $26,22 \%$.

Das gelbe Sulfat zeigte den der erwarteten Formel entsprechenden Schwefelgehalt:

0,1285 Grm. Substanz gaben 0,0472 Grm. $\mathrm{BaSO}_{4}$.

Ber. für $\underset{\mathrm{S}=5,06}{\left(\mathrm{C}_{15} \mathrm{H}_{17} \mathrm{~N}_{5}\right)_{2} \mathrm{H}_{8} \mathrm{SO}_{4}: \quad \text { Gefunden: }} \quad$ 5,05\%.

Die Wirkung der Schwefelsäure dürfte so zu erklären sein dass das Acetylamidrazon zunächst hydrolytisch in Brenztraubensäure (deren Geruch wir deutlich wahrnehmen konnten)ㄱ), Ammoniak und Phenylhydrazin zerlegt wird and dass letzteres dann auf noch unverändertes Acetylamidrazon osazonbildend einwirkt:

1.

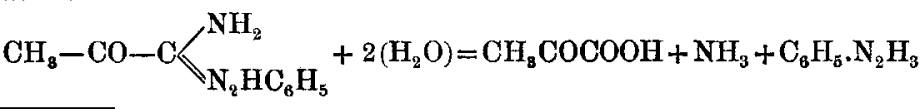

1) Wir konnten die Säure selbst nicht sicher nachweisen, sie scheint bei dem Versuch in Essigsäure und Ameisensäure zu zerfallen. 
242 Bamberger u.deGruyter: Ueb.Formazylmethylketon.

2.

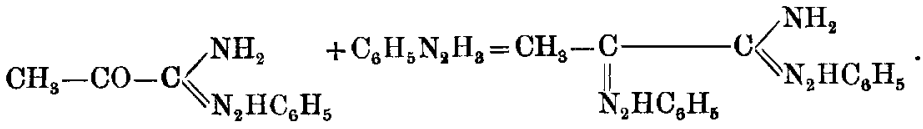

Dass sich Acetylamidrazon thatsächlich in Brenztraubensäure überführen lässt und als Phenylhydrazon ihres Amides anzusprechen ist, geht aus dem folgenden, von Bamberger und Grob ausgeführten Versuch hervor, welcher hier nur ganz kurz erwähnt wird, da er an anderer Stelle genauer beschrieben ist. $\left.{ }^{1}\right)$

\section{Acetylamidrazon und salpetrige Säure.}

Nitrosamin des Brenztraubensäurephenylhydrazids,<smiles>[CH2]OC(O)=NN(C=O)CC</smiles>

Salpetrige Säure wirkt bei Gegenwart von Salzsäure auf Acetylamidrazon nitrosirend und zugleich hydrolytisch spaltend; man erbält neben $1 \mathrm{Mol}$. Ammoniak das in der Ueberschrift bezeichnete Nitrosamin - farblose, glasglänzende, äusserst zersetzliche Nadeln, welche unter Aufschäumen bei $85^{\circ}-85,5^{0}$ schmelzen, sich in Alkalien (einschliesslich Soda und Ammoniak) auflösen und durch Säuren wieder gefällt werden.

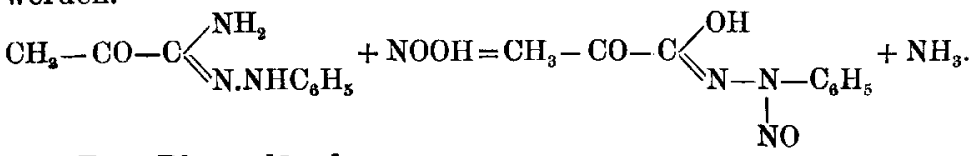

Das Phenylhydrazon,<smiles>CCCCN(C(=O)N=C(C)c1ccccc1)c1ccccc1</smiles>

bildet schwach strohgelbe, fast weisse, seideglänzende Nadeln, rasch erhitzt bei $128^{\circ}-129^{\circ}$ schmelzend und von ausgesprochen sauren Eigenschaften.

Das Paranitrophenylhydrazon,

1) Ber. 34, 539.

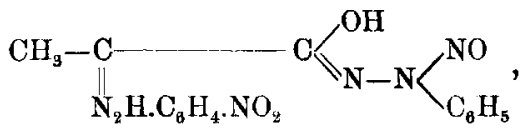


stellt goldglänzende, irisirende Schuppen oder Blättchen vom Schmelzp. $147^{\circ}-148^{\circ}$ dar (Bad auf $135^{\circ}$ vorgeheizt). Mit tief braunrother Farbe in Alkalien löslich.

Durch kochende verdünnte Salzsäure wird das Nitrosamin des Brenztraubensäurephenylhydrazids zur Hauptsache in salpetrige Säure und das bekannte Brenztraubensäurephenylhydrazon ${ }^{1}$ ) zerlegt:

1. $\mathrm{CH}_{3}-\mathrm{CO}-\mathrm{C} \bigotimes_{\mathrm{N}-\mathrm{N}}^{\mathrm{OH}}<_{\mathrm{C}_{6} \mathrm{H}_{5}}^{\mathrm{NO}}+\mathrm{H}_{2} \mathrm{O}=\mathrm{CH}_{3}-\mathrm{CO}-\mathrm{C} \ll_{\mathrm{N} . \mathrm{NHC}_{6} \mathrm{H}_{\mathrm{s}}}^{\mathrm{OH}}+\mathrm{HNO}_{2}$.

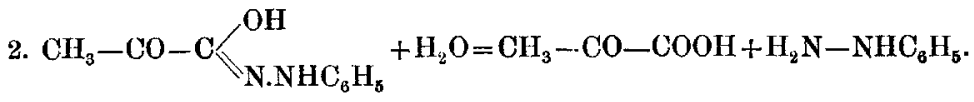
3. $\mathrm{CH}_{3}-\mathrm{CO}-\mathrm{COOH}+\mathrm{H}_{2} \mathrm{~N} \cdot \mathrm{NHC}_{6} \mathrm{H}_{5}=\mathrm{CH}_{3}-\underset{\mathrm{N}_{2} \mathrm{HC}_{6} \mathrm{H}_{5}}{\mathrm{C}-\mathrm{COOH}}+\mathrm{H}_{2} \mathrm{O}$.

Als Nebenprodukte entstehen: Nitrosobenzol, Diazobenzolchlorid, Phenol, Anilin und wahrscheinlich Diazobenzolimid; ausserdem Substanzen unaufgeklärter Natur.

Zum Schluss theilen wir die bisher noch nicht veröffentlichten Analysen einiger dem Formazylmethylketon analoger Verbindungen mit, deren Eigenschaften schon an anderer Stelle $^{2}$ ) mitgetheilt sind.

1) Das Phenylhydrazon des Brenztraubensäureäthylesters,<smiles>CCOC(=O)C(C)(CC)N(CC)CC</smiles>

welches E. Fischer und Jourdan (Ann. Chem. 236, 142) durch Aetherificirung der Säure dargestellt haben, entsteht (beiläufig bemerkt) auch beim Zusatz von Phenylhydrazin zur alkoholischen Lösung des Brenztraubensäureesters. Glasglänzende Säulen vom Sehmelzp. $116^{\circ}-117^{\circ}$ (corr.; F. u. J. $116^{\circ}-117^{\circ}$ ), welche farblos und nicht gelb sind, wie Japp und Klingemann (Ann. Chem. 247, 208) angeben. Analyse:

0,1623 Grm. Substanz gaben 0,3816 Grm. $\mathrm{CO}_{2}$ u. 0,1012 Grm. $\mathrm{H}_{2} \mathrm{O}$.

$$
\begin{array}{cc}
\text { Berechnet für } \mathrm{C}_{11} \mathrm{H}_{14} \mathrm{~N}_{2} \mathrm{O}_{2}: & \text { Gefunden: } \\
\mathrm{C}=64,08 & 64,12 \% \\
\mathrm{H}=6,80 & 6,95 \%
\end{array}
$$

Beim kurzen Kochen mit verdünnter Salzsäure wird der Ester zum Brenztraubensäurephenylhydrazon verseift.

2) Ber. 25, 3546. 
244 Bamberger u.deGruyter: Ueb.Formazylmethylketon.

Bezüglich der Darstellung ist nachzutragen, dass die Lösung der zwei reagirenden Stoffe stets in die eisgekublte Lösung des Natriumacetats bezw: des Natriumcarbonats eingegossen wurde.

$$
\mathrm{CH}_{9}-\mathrm{CO}-\mathrm{C}\left(\begin{array}{l}
\mathrm{N}_{2}-\mathrm{C}_{8} \mathrm{H}_{4}\left(\mathrm{CH}_{8}\right) \text { (p.) } \\
\mathrm{N}_{2} \mathrm{H}-\mathrm{C}_{6} \mathrm{H}_{4}\left(\mathrm{CH}_{3}\right) \text { (p.) }
\end{array} .\right.
$$

$0,1640 \mathrm{Grm}$. Substanz gaben $28,4 \mathrm{Ccm}$. $N$ bei $18^{\circ}$ und $718 \mathrm{Mm}$. B.

Berechnet für $\mathrm{C}_{17} \mathrm{H}_{18} \mathrm{~N}_{4} \mathrm{O}$ :

$$
\begin{aligned}
& \mathrm{N}=19,04 \\
& \mathrm{CH}_{3}-\mathrm{CO}-\mathrm{C} / \mathrm{N}_{2}-\mathrm{C}_{6} \mathrm{H}_{4}\left(\mathrm{NO}_{2}\right)(\mathrm{p} .) \\
& \mathrm{NC}_{6} \mathrm{H}_{5}
\end{aligned}
$$

0,1482 Grm. Substanz gaben 31 Cem. N bei $18^{\circ}$ und $718 \mathrm{Mm}$. B.

Bercchnet für $\mathrm{C}_{15} \mathrm{H}_{13} \mathrm{~N}_{5} \mathrm{O}_{8}$ : Gefunden:

$$
\begin{aligned}
& \mathrm{N}=22,54 \quad 22,79 \% \text {. }
\end{aligned}
$$

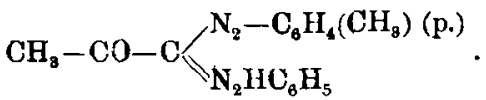

$0,1957 \mathrm{Grm}$. Substanz gaben $37 \mathrm{Ccm}$. $\mathrm{N}$ bei $20^{\circ}$ und $713 \mathrm{Mm}$. B.

Berechnet für $\mathrm{C}_{18} \mathrm{H}_{18} \mathrm{~N}_{4} \mathrm{O}$ : Gefunden:

$$
\mathrm{N}=20,00 \quad 20,21 \% \text {. }
$$

$$
\mathrm{CH}_{8}-\mathrm{CO}-\mathrm{C}\left\langle\begin{array}{l}
\mathrm{N}_{2}-\mathrm{C}_{10} \mathrm{H}_{7}(\alpha) \\
\mathrm{N}_{2} \mathrm{H}-\mathrm{C}_{10} \mathrm{H}_{7}(\alpha)
\end{array} .\right.
$$

0,1497 Grm. Substanz gaben 21,6 Ccm. $N$ bei $17^{\circ}$ und $721 \mathrm{Mm}$. B.

Berechnet für $\mathrm{C}_{28} \mathrm{H}_{18} \mathrm{~N}_{4} \mathrm{O}$ :

$$
\mathbf{N}=\mathbf{1 5 , 3 0}
$$

Gefunden:

$15,81 \%$. 